\title{
Experimental Investigation and High Resolution Simulator of In-Situ Combustion Processes
}

\section{Quarterly Report}

\author{
Start date: January 2004 \\ End date: March 2004 \\ Margot Gerritsen \\ Anthony R. Kovscek \\ April 2004 \\ DE-FC26-03 NT15405
}

\author{
Department of Petroleum Engineering \\ Stanford University \\ Green Earth Sciences Building \\ 367 Panama Street \\ Stanford, CA 94305-2220
}




\section{Disclaimer:}

This report was prepared as an account of work sponsored by an agency of the United States Government. Neither the United States Government nor any agency thereof, nor any of their employees, makes any warranty, express or implied, or assumes any legal liability or responsibility for the accuracy, completeness, or usefulness of any information, apparatus, product, or process disclosed, or represents that its use would not infringe privately owned rights. Reference herein to any specific commercial product, process, or service by trade name, trademark, manufacturer, or otherwise does not necessarily constitute or imply its endorsement, recommendation, or favoring by the United States Government or any agency thereof. The views and opinions of authors expresses herein do no necessarily state or reflect those of the United States Government or any agency thereof.

\section{Abstract}

Accurate simulation of in-situ combustion processes is computationally very challenging because the spatial and temporal scales over which the combustion process takes place are very small. In this first quarterly report of our DoE funded research, we discuss the design of a new simulation tool based on an efficient Cartesian Adaptive Mesh Refinement technique that allows much higher grid densities to be used near typical fronts than current simulators. The formulation presented here for a first one-dimensional simulator will serve as the foundation for the development of a three-dimensional simulator that can handle realistic permeability heterogeneity. The development of the simulation tool will be supported by extensive laboratory experiments conducted to provide validation data, and to study effective variants of the combustion process. The preliminary investigation reported here shows how metallic salt additives can promote and sustain combustion by enhancing the oxidation and cracking of hydrocarbons. 


\section{Table of Contents}

List of graphical materials

1. Introduction

2. Executive Summary

2.1. Personnel

2.2. Important accomplishments

3. Experimental

4. Results and discussion

5. Conclusion

References

\section{List of graphical materials}

Figure 1. Two saturation profiles and the corresponding ACCR grids during an early (left) and later (right) stage of a gas injection process.

Figure 2. BoomerAMG setup and solve time as function of the number of cells in an ACCR grid. 


\section{Introduction}

In-situ combustion, or air injection, is the process of injecting oxygen into oil reservoirs to oxidize the heaviest components of the crude oil and enhance oil recovery through the heat and pressure produced. The emphasis of this work is to study and model numerically in situ combustion processes. The ultimate objectives are to provide a working accurate, parallel in situ combustion numerical simulator and to better understand the in-situ combustion process when using metallic additives and/or solvents combined with in situ combustion. For this purpose, experimental, analytical and numerical studies are conducted.

This report presents results of the second quarter of the first year of this project. 


\section{Executive Summary}

\subsection{Personnel}

Current personnel include Prof. Margot Gerritsen (PI), Prof. Tony Kovscek (Co-PI), Dr. Louis Castanier (Technical manager), Dr. Jonas Nilsson (postdoctoral fellow), Mr. Rami Younis (PhD student) and Mr. Bingjian He (MSc student).

\subsection{Important accomplishments}

\subsubsection{Development of the one-dimensional simulator}

An implementation of the one-dimensional simulator described in Quarterly Report 1 is under development. To date, progress has been made in two areas. A patch-based refinement algorithm for Adaptive Mesh Refinement was implemented and tested for various hyperbolic conservation laws including scalar and low-dimensional systems. The second area is the implementation of a psuedo-equilibrium property value ISC simulator for one-dimensional regular grids. Initial testing highlights difficulties with the current property evaluation approach, and the need for variable substitution methods. Next steps include adapting the code to overcome these issues, and to place it within the AMR framework code, followed by more extensive testing.

\section{AMR implementation}

The patch-refinement approach described in Quarterly Report 1 was implemented to allow for plug-in of almost any partial differential equation solver to act on sub-grids. This has allowed us to test the implementation on more tractable problems with known solution properties. The initial test set included various forms of scalar hyperbolic equations and various discretizations for them. The initial results show that considerable gains in efficiency can be gained without sacrificing accuracy by solving the same problem adaptively rather than by solving it on excessively refined regular grids. While the gains may be considerable however, improvements in the grid hierarchy design algorithms can substantially improve on current efficiency ratios. Moreover, such algorithms naturally tend to require problem specific information, suggesting that they be improved and tailored more specifically to the ISC problem.

\section{ISC simulator}

A simulator for the classical formulation of the ISC three phase, and six component governing equations was developed. Since in such systems, the number of phases present within a given grid-block may vary, the number of unknowns that fix the thermodynamic state of the fluids also varies accordingly. To date, two alternative approaches have been proposed to adapt to such changes. One approach is to assume constant equilibrium coefficients relating fractions of species present in a pair of phases. These constants are modified in such a way that there are three phases present at all times, with the provision that the phase saturations can become very small to model phase disappearance. The second approach first estimates the number of phases that can present, and then modifies the number of variables accordingly.

The current implementation utilizes the first approach. The objective of this implementation is to characterize the robustness and magnitude of sacrifice in accuracy relative to potential gains in computational efficiency. Initial results indicate that when such an approach is applied to more 
realistic relative permeability and thermodynamic property descriptions, even slight pseudoconstant modifications can deter the algorithm from converging for regions with sharp phase variations.

\subsubsection{Extension of the simulator to three dimensions}

(This part of the research is also reported in [1])

In 3D, we have implemented an Anisotropic Cartesian Cell-based Refinement (ACCR) technique, based on the work by Ham et al. [2]. This is the first time this technique is applied to petroleum engineering in general and in-situ combustion processes in particular. The anisotropic refinement is a natural approach because of the typically highly structured nature of reservoir heterogeneity. The grid is formed by a number of anisotropic refinements of its cells, i.e. splitting a cell in half, to construct a suitably refined mesh. One obvious advantage of the method is its simplicity and generality; wherever refinement is needed, grid cells are refined. Furthermore, the efficiency rivals unstructured methods because of the underlying Cartesian grid structure and it allows us to resolve the narrow zones locally without refining the entire domain. Figure 1 shows two ACCR grids for saturation profiles in a gas injection process. The detection algorithm is based on gradients and an approximate threshold.

A novel higher order discretization of the elliptic Pressure equation has been developed on these grids. This is relatively straight forward on uniform Cartesian grids, the anisotropically refined cells makes it more difficult. To obtain a formally second-order accurate method, the pressure equation is discretized by a compact finite volume method. The fluxes are computed by a threepoint finite difference approximation combined with a cubic interpolation of the cell centered pressure values in the adjacent discretization cells. The simulator is parallelized using the Message Passing Interface (MPI) for distributed memory machines.

The resulting system of linear equations is solved by the parallel algebraic multigrid solver BoomerAMG [3]. The coarsening is done by the Falgot technique which is a combination of Cleary-Luby-Jones-Plassman coarsening and the classic Ruge-Stueben coarsening algorithm. For the relaxation a hybrid Gauss-Seidel /Jacobi scheme is used. Some preliminary results shows linear convergence for the pressure solve when we increase the number of cells for a highly anisotropic Cartesian 3D grid, see Figure 2.

\subsubsection{Experimental in-situ combustion}

Experiments are underway in a kinetics cell to understand the role that metallic additives, here salts of some common metals, play in improving the in-situ combustion process. They improve combustion by affecting cracking, low temperature oxidation, and pyrolysis reactions leading to fuel lay down during in-situ combustion. The mechanism by which metallic additive catalyze combustion reactions is not yet known. Developing a mechanistic understanding is one key to improving and expanding application of in-situ combustion.

It is our hypothesis that the cationic metals ion exchange with clay ions creating an activated site that acts in a manner analogous to a heterogeneous catalyst. An experimental program is underway to test this hypothesis. The experiment apparatus is described in section 3.

The experimental plan is to measure baseline kinetics of the crude oils. These systems contain oil, water, sand, and kaolinite clay material. Then the clay is replaced with a fine-grained, inert silica 
powder. The silica powder provides surface area, but has no activated surface sites. Third, the sand, clay, oil mixture is doped with metallic additive, Fourth, the sand, silica powder, oil mixture is doped with metallic additive. Each of these mixes is then subjected to measurement of its reaction kinetics. Table 1 summarizes results to date.

In the two runs with silica powder instead of the clay kaolinite, the experiments using $\mathrm{Fe} 3+$ and Cymric light crude oil showed an oxygen consumption peak that increased by $21 \%$ for LTO reaction. The activation energies decreased by $5 \%$ and $8 \%$ for LTO and HTO, respectively. In another two runs with kaolinite, the catalytic effect of $\mathrm{Fe} 3+$ is strikingly enhanced by the existence of kaolinite. The peak consumption of oxygen during LTO for the run with $\mathrm{Fe} 3+$ is $5.1 \%$, while that of the run without $\mathrm{Fe} 3+$ is just 3\%, an increment of $67 \%$. The oxygen consumption peak for HTO increased by $36 \%$ for the run with $\mathrm{Fe} 3+$. Compared the pair with kaolinite with the pair with silica, it is obvious that the kaolinite greatly enhanced the catalytic effect of the metallic additive. Kaolinte itself has some Fe3+. But the role of kaolinte is enlarged by enhancing the catalytic effect of $\mathrm{Fe} 3+$.

Cymric heavy oil behaved in a fashion similar to the runs on light oil. The catalytic effect of $\mathrm{Fe} 3+$ is not obvious on the system using silica, comparing the oxygen consumption and activation energy. While in the runs with kaolinite, the catalytic effect of $\mathrm{Fe} 3+$ is encouraging, especially in the performance of HTO reaction. Oxygen consumption picked up from $12.2 \%$ for the run without $\mathrm{Fe} 3+$ to $18.4 \%$ for the run with $\mathrm{Fe} 3+$, an increment of $51 \%$. The increment for LTO reached only $8.7 \%$. The same trend is observed in the change of activation energy.

It should also be noted that the catalytic effect of light oil concentrated on the LTO, while that of heavy oil concentrated on the HTO.

\section{Experimental}

The experiment apparatus consists of a kinetics cell, gas analyzer, and data logging system. Oxygen and nitrogen were provided by gas cylinder and metered using a mass flow controller. The kinetics cell consisted of a thick-walled stainless steel cylinder with length of $5.25 \mathrm{in}$. and outside diameter of $1.9 \mathrm{in}$. On the top of the cylinder is the tube assembly that allows air injection and a port for temperature measurements. The cell is packed with a mixture of oil, water, sand, clay or a fine-grained high surface area silica, and aqueous metallic additive, in some cases. The oils tested so far have included: a paraffinic white mineral oil, decane $\left(\mathrm{C}_{10}\right)$, a long chain alkane $\left(\mathrm{C}_{30}\right)$, light $\left(34^{\circ} \mathrm{API}\right)$ crude oil from Cymric $(\mathrm{CA})$, and heavy $\left(12^{\circ} \mathrm{API}\right)$ crude oil from Cymric (CA). Most of our work has been on the light and heavy crude oils. The metallic additive is a salt of iron $\left(\mathrm{Fe}^{3+}\right)$.

\section{Results and discussion}

This report covers the second quarter of our research grant. The quarter was used primarily to further design the basic computational algorithms, and to establish the methodology for our experimental work. We have made very good progress in these areas again this quarter, and have submitted a second paper on our work on the three-dimensional extension in [3].

\section{Numerical simulations}

We are gaining confidence in the choice of our numerical approaches. The preliminary results shown in figures 1 and 2 for the 3D extension are especially encouraging. As stated in the 
introduction, the simulation of in-situ combustion processes requires a very high level of grid refinement. The Anisotropic Cartesian Cell-based Refinement (ACCR) approach allows a very localized refinement and very fast transitions between fine and coarse grid blocks. This can clearly be seen in figure 1 . This minimizes the number of grid points required, which is especially attractive in ISC processes since the work per grid block is high (thermodynamic equations).

The current multi-grid implementation is more efficient than we had originally expected. We do strongly feel that a Full Approximation Scheme (FAS), a variant of Geometric MultiGrid, is ideally suited for our application. We will therefore start the design of our FAS scheme shortly.

\section{Experimental work}

As discussed in section 2, the deposition of fuel is a decisive factor for successful combustion. If oil is too light, insufficient fuel is deposited and it is not enough to sustain combustion. For heavier oils, too much fuel deposition leads to a greater requirement for air.

Kaolinite and metallic additives both have catalytic effect on combustion performance. Furthermore, the effect of metallic additives is enhanced by kaolinite. Clay has an obvious effect on crude combustion, one is via physical perspective, and the other is chemical one. Physically, clay has large external and internal surface area that dramatically improves the deposition of fuel on the sand matrix and constitutes a strong carrier for various reactions in different stages of the combustion. Chemically, the reaction of clay (kaolinite in our experiment) during in situ combustion is divided into two categories; these two categories are not exclusive, but interactive. One is the reaction of clay with organic materials in crude oil; the another is that with the metallic additives.

The catalytic effect varied for different oils. For Cymric light oil, the catalytic effect is obvious in LTO reaction, while for Cymric heavy oil, the HTO reaction shows the greatest catalytic effect.

\section{Conclusions}

It is still too early in the numerical algorithm development to draw sound conclusions from our preliminary investigations. For example, our 1D simulation does not currently have an accurate way of dealing with appearing and disappearing phases. Also, we are still researching a suitable multi-physics approach to deal with the very computationally challenging kinetics. However, we are developing ideas and hope to report on a few novel approaches in the next quarterly report. A very positive outcome of the research done in this quarter is the development of the novel second order accurate pressure scheme for ACCR grids. We will be extending this in the next two quarters to heterogeneous media.

On the experimental side, it is apparent that metallic additives improve combustion of light oil. Without additives, some light oils (such as Cymric) could not sustain combustion. We have also found that the deposition of fuel is a decisive factor for successful combustion. If oil is too light, insufficient fuel is deposited and it is not enough to sustain combustion. Fore heavier oils, too much fuel deposition leads to a greater requirement for air.

We will focus in the future on understanding how the additives work during combustion. In the near future we hope to obtain a sample of crude oil from Ugnu (AK) so that kinetics of reactions can be determined similarly as well the effect of metallic additives tested. The test well from where we had hoped to obtain a sample is currently shut in because the pump had sanded up. After cleaning, the operator hopes to obtain at least a gallon of oil for kinetics measurements. 
We would like to add that responses from industry and academic colleagues so far has been very positive, which was again emphasized in our annual Affiliates meeting in May 2004.

\section{References}

[1] J. Nilsson, M. Gerritsen and R. Younis, Parallel Anisotropic Cartesian Grid Adaptation for In-Situ Combustion Simulations, 9th European Conference on the Mathematics of Oil Recovery, Cannes

[2] F. Ham, F. Lien and A. Strong, A Cartesian Grid Method with Transient Anisotropic Adaptation, Journal of Computational Physics, Volume 179, Issue 2, 1 July 2002, Pages 469-494.

[3] V. Henson and U. Yang, BoomerAMG: a Parallel Algebraic Multigrid Solver and Preconditioner, Applied Numerical Mathematics, 41, 155--177, 2002. 

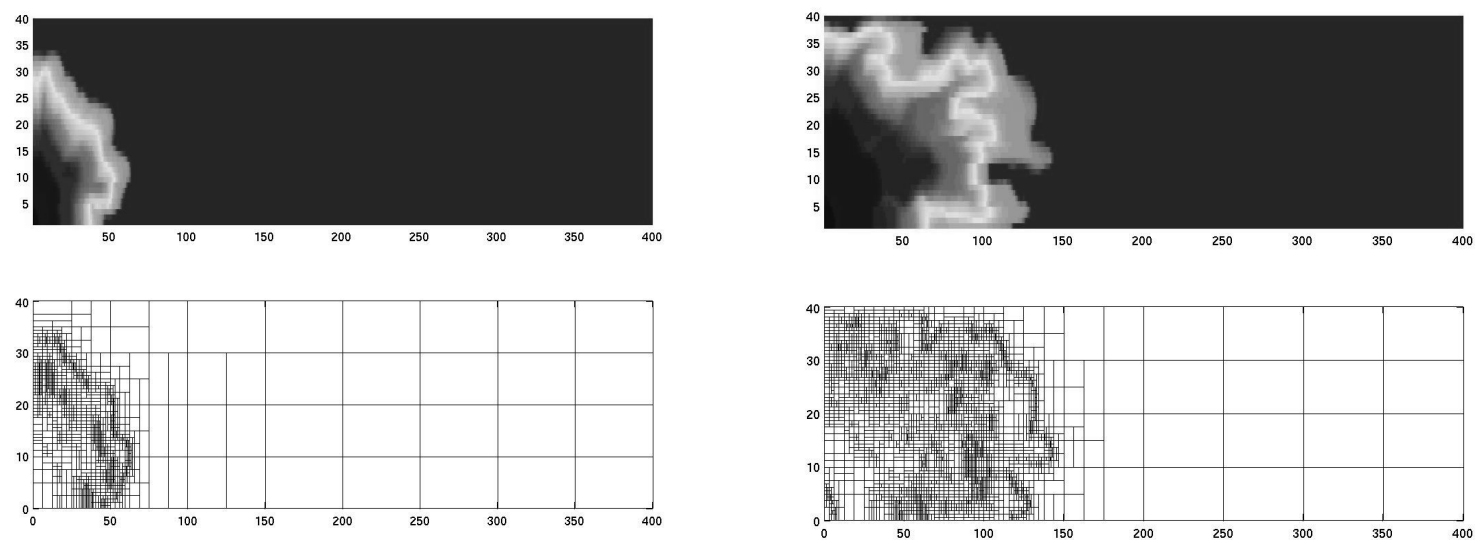

Figure 1. Two saturation profiles and the corresponding ACCR grids during an early (left) and later (right) stage of a gas injection process. Note the very local refinements near saturation fronts.

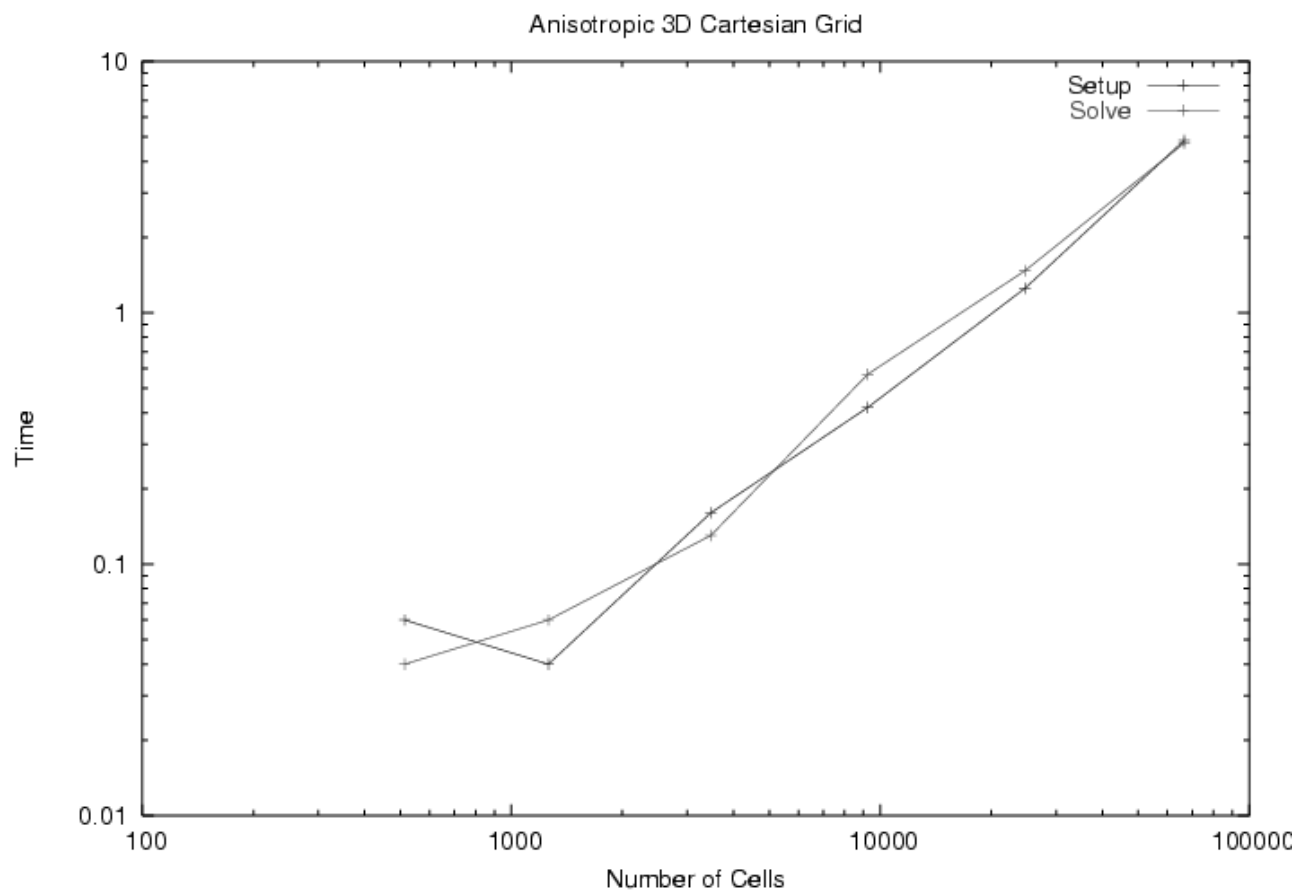

Figure 2. BoomerAMG setup and solve time as function of the number of cells in an ACCR grid. Note the linear relation between solve time and number of cells. 
